\title{
Objects and their locations in exogenous cuing
}

\author{
KRISTA L. SCHENDEL \\ University of California, Davis, California \\ LYNN C. ROBERTSON \\ University of California, Berkeley, California \\ and Veterans Affairs, Martinez, California \\ and \\ ANNE TREISMAN \\ Princeton University, Princeton, New Jersey
}

\begin{abstract}
Anatomical, physiological, and behavioral studies provide support for separate object- and locationbased components of visual attention. Although studies of object-based components have usually involved voluntary attention, more recent evidence has suggested that objects may play an independent role in reflexive exogenous orienting, at least at long stimulus onset asynchronies (SOAs). In the present experiments, the role of objects in reflexive attentional orienting was investigated by developing a task in which location and object cuing could be separately examined for both short and long SOAs. Typical location cuing effects were obtained, indicating facilitation at short cue-targetintervals and inhibition of return (IOR) at longer intervals. In contrast, object cuing resulted in facilitation for cued objects at long cue-target intervals and no object-based IOR. Interestingly, object cuing primarily affected targets at cued locations, and not those at uncued locations. Together, the experiments examine the interactive nature of objects and locations in exogenous orienting and seem most consistent with a location-mediated view of object-based orienting.
\end{abstract}

Studies of visual attention (e.g., Posner \& Cohen, 1984; Tipper, Weaver, Jerreat, \& Burak, 1994; Treisman \& Gelade, 1980; Yantis \& Hillstrom, 1994) have shown that attention plays a vital role in perceptual processing. An increasing number of studies have also begun to uncover relationships between behaviorally identified attentional mechanisms and their underlying neural substrates (Connor, Preddie, Gallant, \& Van Essen, 1997; Corbetta, Miezin, Dobmeyer, Shulman, \& Petersen, 1991; Mangun, 1995; Mangun, Hopfinger, Kussmaul, Fletcher, \& Heinze 1997; Moran \& Desimone, 1985; Olson \& Gettner, 1995). These neurobiological studies have provided evidence that attention modulates perceptual processing at various levels in the visual system. Yet, how and when attention is directed remains a topic of investigation.

Behavioral studies have contributed extensively toward an understanding of attention by exploring which aspects of the visual environment are selected. For instance, evidence has accumulated in support of object-based mechanisms of attention in addition to space-based mechanisms (Behrmann, Zemel, \& Mozer, 1998; Duncan, 1984; Egly,

Portions of this research were presented in poster format at the Annual Cognitive Neuroscience Society Meeting held in Boston, April 1999, and in a paper presentation at the Cognitive Science Association for Interdisciplinary Learning (CSAIL), July 1998. The research was supported by NSF Grant SBR-9222118 to the second and third authors and by a Floyd and Mary Schwall Fellowship awarded to the first author. Correspondence regarding this article should be addressed to K. L. Schendel, Neurology Research 127E, Veterans Administration, 150 Muir Road, Martinez, CA 94553 (e-mail: kristals@ebire.org).
Driver, \& Rafal, 1994; Jordan \& Tipper, 1998a, 1998b; Marshall \& Halligan, 1993; Moore, Yantis, \& Vaughan, 1998; Tipper \& Behrmann, 1996; Tipper \& Weaver, 1998; Treisman, Kahneman, \& Burkell, 1983). An understanding of the degree to which these two types of attention may be separate or complementary will ultimately aid our understanding of the neural circuitry and cognitive architecture of visual attention. However, achieving this understanding has been difficult (see Baylis \& Driver, 1993; Buxbaum, Coslett, Montgomery, \& Farah, 1996; Kim \& Cave, 1995; Kramer, Weber, \& Watson, 1997; Vecera, 1994, 1997; Vecera \& Farah, 1994).

\section{The Where/What Distinction}

Neurobiological evidence in support of a distinction between object and location processing in the visual system comes from studies of brain-damaged patients and anatomical studies in monkeys. Investigations of neurological patients have revealed compelling dissociations between object localization and object identification abilities (Farah, 1990; Robertson, Treisman, Friedman-Hill, $\&$ Grabowecky, 1997). The neural basis of these dissociations has been attributed to complementary pathways for visual analysis-namely, a dorsal where pathway that is important for analyzing the location and movement of visual stimuli and a ventral what pathway that is more involved in object identification (Mishkin, Ungerleider, \& Macko, 1983; Ungerleider \& Haxby, 1994).

A similar distinction appears in the attention literature in the debate between attentional selection of objects and 
locations. Since there appear to be two complementary perceptual pathways, it is likely that both forms of attention play a role in perception. In fact, both object- and space-based attentional deficits have been shown in patients with unilateral neglect (Driver, 1995; Marshall \& Halligan, 1993; Tipper \& Behrmann, 1996).

\section{Spatial Attention}

Spatial attention has been compared with a spotlight or a zoom-lens that can be directed toward particular locations in space. Much of the evidence supporting such location-based models of attention has come from spatial cuing paradigms (Eriksen \& St. James, 1986; Maylor, 1985; Müller \& Rabbitt, 1989; Posner \& Cohen, 1984; Posner, Snyder, \& Davidson, 1980). In these paradigms, participants are required to detect or identify targets presented at peripheral locations, and the effects of attentional cuing are measured by comparing reaction times (RTs) or accuracy for targets appearing in cued versus uncued locations. Typically, performance is better at cued locations than at uncued locations, but the pattern varies depending on the type of cue and the cue-to-target delay, or stimulus onset asynchrony (SOA).

With central symbolic cues that are predictive of target location (e.g., arrows), faster RTs are observed at cued locations, asymptoting approximately $300 \mathrm{msec}$ post cue onset (Müller \& Rabbitt, 1989; Posner et al., 1980). This facilitation is taken to reflect endogenous attention, or the volitional allocation of attention to the cued location. In contrast, studies of exogenous attention, which will be the focus of this report, tap into more reflexive or automatic mechanisms and, in the pure case, rely on nonpredictive peripheral cues (such as luminance changes). Although such cues provide no information about the upcoming target, they tend to produce two separate effects at the cued location (Posner et al., 1980). At short SOAs there is a facilitatory effect, which is believed to reflect the reflexive summoning of attention to the cue, and at long SOAs there is an inhibitory effect that has been termed inhibition of return (IOR; Maylor, 1985; Maylor \& Hockey, 1985; Posner \& Cohen, 1984; Posner, Rafal, Choate, \& Vaughan, 1985). Tassinari, Aglioto, Chelazzi, Peru, and Berlucchi (1994) have reported data suggesting that the facilitatory and inhibitory components of exogenous orienting are independent, albeit additive, processes (see also Ro \& Rafal, 1999).

\section{Object-Based Attention}

There is clear evidence that attention can also be influenced by objects (Behrmann \& Tipper, 1999; Duncan, 1984; Egly et al., 1994; Gibson \& Egeth, 1994; Robertson \& Kim, 1999; Tipper et al., 1994). To disentangle reflexive orienting to objects and to locations, Egly et al. used displays that contained two rectangles. Targets could appear in either end of the two rectangles, resulting in four possible target locations positioned equidistant from one another. On each trial, a cue that was $75 \%$ predictive of target location appeared at one of the four locations. Uncued targets could be presented either in the opposite end of the cued object or in the uncued object, equidistant from the cued location. Cued targets were facilitated relative to both types of uncued targets, but an extra cost was found for shifting attention between objects, as compared with a spatially equivalent shift within an object. Egly et al. concluded that attention can be allocated to objects as well as to locations, at least under conditions of predictive cuing. Attempts to replicate these findings with nonpredictive exogenous cues have produced mixed results (Barnes, Robertson, Beuche, \& Kim, 1999; Macquistan, 1997).

Thus far, it is unclear whether the distinction between exogenous and endogenous attention that emerges clearly in research on spatial orienting is also present in object-based orienting. Are attentional resources reflexively allocated to objects as well as locations, or are objects selected for attention only at later stages of processing? To date, most demonstrations of object-based attention have relied on tasks that involve some component of endogenous orienting (Duncan, 1984; Egly et al., 1994; Moore et al., 1998; Robertson \& Kim, 1999; Vecera \& Farah, 1994). Here, we will focus on the role of objects in exogenous orienting.

A second issue of theoretical importance is whether objects are attended independently of their locations (pure object hypothesis) or merely seem to be attended because attention is guided toward or away from the locations they occupy (location-mediated hypothesis). According to the former view, attention can select an object on the basis of features other than location, whereas the latter view suggests that an object's location is critical for attentional selection.

The location-mediated hypothesis has received support from the observation that the extra cost for shifting attention between objects in the Egly et al. (1994) paradigm can be modulated by varying the spatial separation between them (Vecera, 1994). Further evidence comes from a visual search paradigm. Kim and Cave (1995) compared RTs to a post-stimulus probe that appeared in a previous target location, a previous distractor location, or a blank location. Responses to the probe were faster in target locations and slower in distractor locations than in blank ones. If the target attracted attention and the distractors were inhibited (Maljkovic \& Nakayama, 1994), the results are consistent with lingering effects of attention at the corresponding locations. This implies that the role of objects in visual attention is to define which locations should or should not be attended.

However, it has been argued that under the right conditions, attention can select object representations independently of their locations (Duncan, 1984; Vecera, 1997; Vecera \& Farah, 1994). In contrast to the findings from the Egly et al. (1994) paradigm, Vecera and Farah found that costs for shifting attention between two overlapping objects in a feature-report task were unaffected when the objects were spatially separated. These findings suggest that object-based selection may indeed operate independently of location-based selection. Still, several challenges have been leveled at the notion that attention is allocated to spatially invariant object representations (see Baylis \& Driver, 1993; Kramer et al., 1997; Vecera, 1994). Most of these challenges are specific to experiments using 
superimposed objects. The present experiments provide a means of disentangling objects from their locations, without manipulating the objects' spatial properties.

\section{Dissociating Objects and Locations}

One way to separate objects and locations is to move objects subsequent to cuing. The question is whether attention will follow the objects or remain tied to the cued location. Using this approach with nonpredictive (exogenous) precues, Tipper et al. (1994) were able to compare attentional orienting to cued versus uncued objects, neither of which occupied a cued location. The results indicated that the inhibitory component of exogenous orienting (IOR) followed the cued object to its new location, suggesting that attention was exogenously allocated to the cued object. However, an alternative interpretation is possible: Note that the objects in Tipper et al.'s experiment retained their relative locations as they rotated around the fixation point. Thus, the objects may have set up a frame of reference in which attentional orienting operated not on objects, but on relative as opposed to absolute locations. This would be consistent with Baylis and Driver's (1993) conception of hierarchical location coding. ${ }^{1}$

Another method for dissociating the role of objects and locations in attentional orienting was used by Jordan and Tipper (1998b). They examined attention effects in the presence or absence of illusory objects. Large arrays of pacmen stimuli were arranged so that illusory (Kanizsa) squares appeared along one axis of the display, whereas pacmen along the orthogonal axis were oriented so as not to form illusory objects. IOR was observed at all cued locations but was greater for locations occupied by an illusory object. However, in these cluttered displays, it is difficult to know whether the increase in IOR was due to the presence of an apparent object or the presence of illusory contours that better defined candidate locations. In fact, for uncued baseline trials, the authors did report a trend for target detection to be faster when the target was presented within an illusory object. Although they conducted a control experiment to confirm that cue detection was not facilitated by the presence of the Kanizsa contours, they did not conduct the same experiment for targets in the absence of cues, leaving open the possibility that target detectability varied across the two conditions.

Most studies in which the role of objects in reflexive orienting has been examined have focused on IOR because stimulus preparation (either motion or the generation of illusory objects) takes longer than the SOA at which facilitation is normally observed (see Ro \& Rafal, 1999, for an exception). However, if objects play a role in exogenous orienting, we might expect both facilitatory and inhibitory effects. In the experiments to be reported, stationary objects were used, allowing both short and long SOAs to be investigated.

The specific goal of this study was to compare and contrast, within the same experimental paradigm, the facilitatory and inhibitory components of location- and objectbased mechanisms in exogenous orienting. In order to separate the effects of cuing an object from those of cuing its location, we manipulated the shape of the location markers in a Posner cuing task. If attention can be allocated to locations independently of the objects that occupy them, changing the shape of the location markers should have little or no effect on the usual pattern of exogenous spatial orienting. Such a finding would strengthen views that exogenous attention is primarily spatial. However, if reflexive attention can be allocated to objects independently of their locations, we should observe early facilitation and/or later inhibition of cued relative to uncued objects when location cuing is held constant. Such a result would suggest that exogenous attention can be allocated to objects independently of their locations, or to spatially invariant object representations. In the event that both spatial and object-based effects are observed, examination of the interaction and respective timecourse of object and location cuing will help us to understand the cumulative effects of directing attention within displays containing multiple objects. Finally, if the primary role of objects is to guide attention to particular locations (location-mediated hypothesis), an interaction between object and location cuing should be observed even in the absence of dominant object-based effects.

\section{EXPERIMENT 1}

In order to separate object cuing from location cuing, we used Star of David stimuli, composed of two overlapping triangles, as the location markers. The star-shaped markers could remain as stars or change into triangles through the offset of one component triangle. Location markers that changed in shape following the cue were operationally defined as uncued objects, whereas those that remained the same were defined as cued objects. This allowed us to test not only cued and uncued locations, but also cued and uncued objects within the same experimental paradigm. If exogenous attention is allocated to particular objects, changing the shape of the cued object should reduce the effects of cuing. On the other hand, if attention is allocated to locations and the primary role of objects is to mark spatial locations, location marker shape should be irrelevant.

Other research (Schendel, Robertson, \& Treisman, 2001) has shown greater IOR effects following a unilateral offset in the display, as compared with displays with an offset on both sides. In order to minimize the effects of this extraneous factor, the shape of the uncued location marker was always changed (via partial offset), ensuring a bilateral visual offset on every trial. It could be questioned whether the cue offset on the one side was adequately balanced by a partial object offset on the opposite side. However, RTs to targets appearing opposite a shape change did not differ from those in the same cuing condition that appeared opposite a mere cue offset (see the Results section, below).

To isolate the effects of location cuing, we compared target detection RTs in cued and uncued locations when both location markers changed shape immediately after the presentation of the cue, becoming uncued objects. This allowed us to compare responses to cued and uncued 


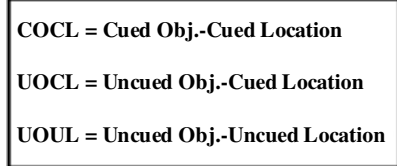

\section{Both Markers Change}

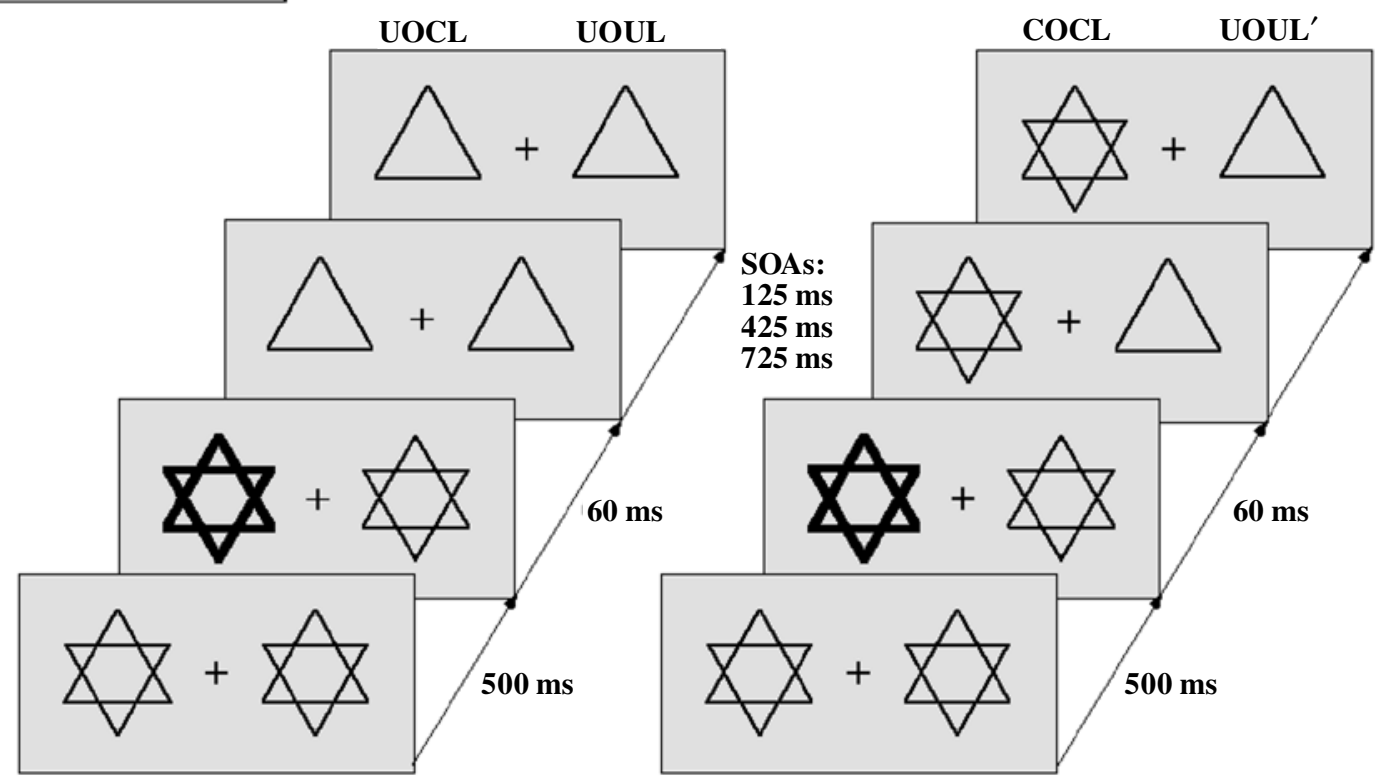

\section{Uncued Marker Changes}

Figure 1. All stimulus sequences in Experiment 1 involved bilateral offsets. This required a partial offset of the uncued marker on every trial (as cue offsets on one side, part of the marker offsets on the opposite side). Targets (\#) appeared with equal probability in one location or the other.

locations in the absence of any cued objects (UOCL vs. UOUL; see Figure 1).

To isolate the effects of object cuing, we compared target detection RTs in cued and uncued objects at the cued location. On uncued object trials, the marker at the cued location changed from a star to a triangle. On cued object trials, the marker at the cued location remained unchanged (as a star) when the cue offset. Thus, the critical comparison involved target detection at the cued location when the target appeared either in a cued object (star, COCL) or in an uncued object (triangle, UOCL).

\section{Method}

\section{Participants}

After giving informed consent, 12 University of California, Davis undergraduates ( 7 female, 5 male) participated in the study for course credit. All participants were naive as to the purpose of the experiment and reported normal or corrected-to-norm al vision. Three were left-handed.

\section{Stimuli and Apparatus}

The stimuli were presented on a Macintosh 16-in. color monitor in black on a light gray background. The initial stimulus display consisted of a central fixation + and two Star of David outlines. Each Star of David stimulus was created by superimposing one downward- and one upward-pointing triangle. At a viewing distance of approximately $60 \mathrm{~cm}$, the stars subtended $2.5^{\circ} \times 2.1^{\circ}$ of visual angle and were centered at $6^{\circ}$ to the left and right of fixation. The distance between the inner edge of each star and fixation was $4.25^{\circ}$. Cuing was implemented by thickening all the lines of one of the stars from $0.07^{\circ}$ to $0.13^{\circ}$. Star of David stimuli were changed into triangles via the disappearance of one component triangle. Individual triangles each subtended $1.8^{\circ} \times 2.1^{\circ}$ of visual angle. Targets (\#) were presented in bold Helvetica font size 34 and appeared in the center of one of the locations initially defined by the stars. A computer interface box was used to allow buttonpresses to be made on an external response box and recorded by the computer.

A standard chinrest restricted head movement throughout the experiment. Eye movements were monitored via a Pulnix camera lens mounted on top of the display monitor that projected an image of the participant's eyes to an additional monitor visible only to the experimenter. Trials containing eye movements were marked by experimenter keypress and excluded from further analysis. Criteria for exclusion included any observable shift of the eyes between the warning beep at the beginning of each trial and the participant's manual response. With this technique, eye movements of approximately $2^{\circ}$ or more are easily detected, whereas smaller ones may be missed. Thus, as a further precaution, all peripheral stimuli were presented $6^{\circ}$ from fixation, and cues and targets never exceeded $100 \mathrm{msec}$ in duration. Trials with eye movements were not replaced but were few in number.

\section{Procedure}

The participants were instructed to press a button as rapidly as possible whenever they saw the target. They were told that targets would appear in one of the two marked locations and that the cues were not predictive of the location or timing of the forthcoming target. It was explained that the stars would sometimes change in shape but that this was irrelevant to the task. The participants were given a practice block of 20 trials, which served to introduce them 
to the display and get them used to maintaining central fixation. All responded with their dominant hand.

Each trial began with a soft beep followed by the presentation of the fixation cross and two Stars of David, one to the left and one to the right of fixation, for $500 \mathrm{msec}$. After the initial display, one of the two stars was cued (thickened) for $60 \mathrm{msec}$. In half of the trials, one component triangle from each star disappeared simultaneously with the removal of the cue, leaving the fixation cross and two triangles (uncued objects), one at the cued location and one at the uncued location. The triangles pointed up or down with equal probability but always pointed in the same direction. In the remaining trials, only the star at the uncued location changed in shape when the cue offset, leaving a cued object (star) in the cued location and an uncued object (triangle) in the uncued location. Again, the triangle was equally likely to be pointing up or down. Thus, in half of the trials both location markers changed shape when the cue offset, and in half of the trials only the uncued location marker changed shape (see Figure 1). For both trial types, targets appeared with equal likelihood in either the cued location or the uncued location following a variable cue-totarget SOA of 125, 425, or $725 \mathrm{msec}$. The targets were presented for $100 \mathrm{msec}$, whereas the fixation cross and location markers remained on the screen until a response was made or until $2.25 \mathrm{sec}$ had elapsed.

As has been explained above, the uncued location marker always changed in shape. As a result, we did not test the condition in which targets appeared in a cued object at the uncued location (COUL). However, we did test two different versions of the UOUL condition. In one case, both location markers in the display changed shape (UOUL), whereas in the other case (denoted UOUL'), only the uncued location marker changed in shape. This allowed us to examine how a shape change opposite an otherwise identical target affected performance.

Twenty-five percent of the targets appeared in a COCL, $25 \%$ appeared in an uncued object at a cued location (UOCL), 25\% appeared in an uncued object at an uncued location (UOUL), and another $25 \%$ also appeared in an uncued object at an uncued location with the difference that the opposite object remained unchanged $\left(\mathrm{UOUL}^{\prime}\right){ }^{2}$

Trial type (both markers change shape vs. uncued marker changes shape) and SOA were randomly varied across trials, with the constraint that no more than three trials in a row would contain the same trial type or SOA. Target validity (i.e., the appearance of the target in a cued location vs. an uncued location) and target visual field were pseudorandomly varied, with no more than four trials in a row containing a target of the same cuing status or the same visual field, respectively.

Approximately $11 \%$ of the trials were catch trials, which contained no target. Overall, each experimental session consisted of 48 catch trials, 192 cued targets, and 192 uncued targets, for a total of 432 trials. The participants were given a short break after every 72 trials, and each participant completed one experimental session.

\section{Results}

In this and all subsequent analyses, outliers and anticipatory responses were removed from the data sets prior to analysis. Outliers were defined as RTs more than three standard deviations from the mean for each individual participant. Anticipatory responses were defined as responses faster than $150 \mathrm{msec}$. This resulted in the removal of fewer than $2 \%$ of all observations. The main interest was in the RTs, since the error rates, given below, were low and did not vary across conditions.

\section{Response Time}

A $2 \times 2 \times 3$ analysis of variance (ANOVA) was performed on the trimmed means, with trial type (both markers change shape or uncued marker changes shape), location cuing (cued or uncued), and cue-to-target SOA $(125,425$, or $725 \mathrm{msec})$ as within-subjects factors.

A main effect of location cuing $[F(1,11)=6.26, p<$ .03 ] showed that the participants responded significantly faster to targets in cued locations than in uncued locations. Given that two of the three SOAs tested in this experiment fall within the range in which IOR is typically observed, an overall benefit for cued locations is somewhat surprising. However, low levels of IOR, possibly owing to a tradeoff at longer SOAs between the inhibition of cued locations and attention to cued objects, could explain this observation. This possibility is discussed below.

A significant main effect of SOA $[F(2,22)=9.59, p<$ $.01]$ reflected slower RTs at the 125-msec SOA $(M=$ $430 \mathrm{msec}$ ), suggesting that RTs decreased with longer cue-to-target intervals. There was no main effect of trial type on target detection RT $[F(1,11)=3.38, p=.09]$, providing no evidence that RTs were differentially affected by unilateral versus bilateral shape changes in the display. The near identity of the results in the UOUL and UOUL' conditions across SOA further confirmed this insensitivity to unilateral shape changes. Target detection was equivalent whether or not there was a shape change on the opposite side of the display. Provided that some kind of visual offset was present on both sides of the display, a unilateral shape change was no more or less distracting than shape changes that occurred bilaterally.

Since our main interest was in the comparison of the effects of location and of object cuing, we performed three different ANOVAs on restricted data sets, each shown in Figure $2 .^{3}$

Location cuing. First, we isolated the effect of location cuing by analyzing all trials in which both markers changed shape after cuing producing only uncued objects (UOCL vs. UOUL). The factors were location cuing and SOA. As is shown in Figure 2 (top left), location cuing interacted with SOA $[F(2,22)=10.58, p<$ $.001]$, showing significant facilitation of the cued location at the short SOA $[F(1,22)=9.47, p<.01]$, and significant IOR at the longest SOA $[F(1,22)=11.7, p<$ $.01]$. Thus, typical location cuing effects were obtained despite shape changes in the location markers that occupied those locations.

Object cuing. Next, we examined the effects of object cuing while holding location cuing constant (Figure 2, top middle). In order to do so, we took all the trials in which targets were presented at a cued location and then compared the targets that had appeared in cued objects with those that had appeared in uncued objects (COCL vs. UOCL). The factors were object cuing and SOA. A significant main effect of object cuing indicated a benefit for cued objects, as compared with uncued objects $[F(1,11)=6.86, p<.05] .{ }^{4}$ However, with this shape-based definition of object cuing, no object-based IOR was observed. A significant object cuing $\times$ SOA interaction $[F(2,22)=4.38, p<.05]$ confirmed that the advantage for cued objects was restricted to the 725 - 

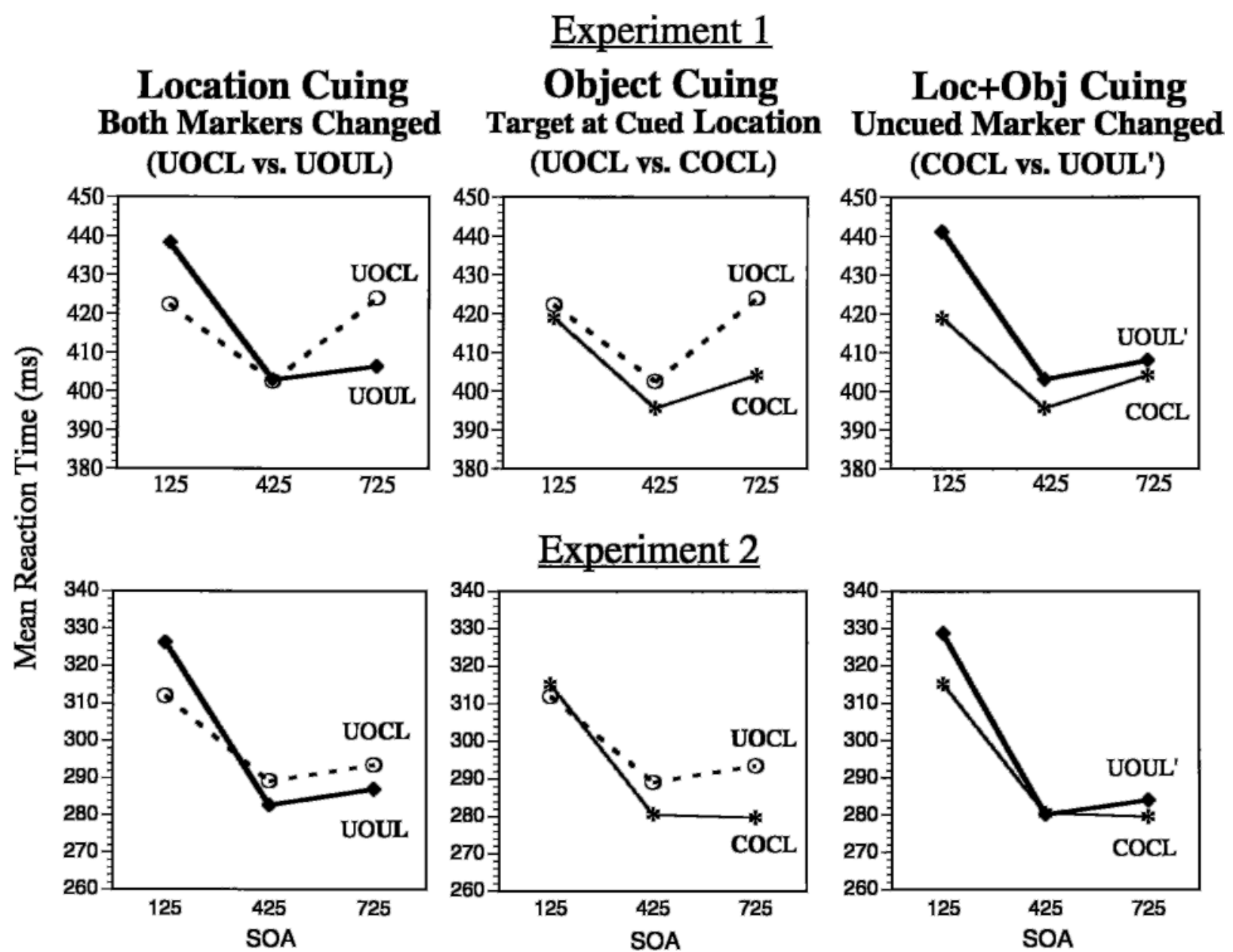

\section{Experiment 2}
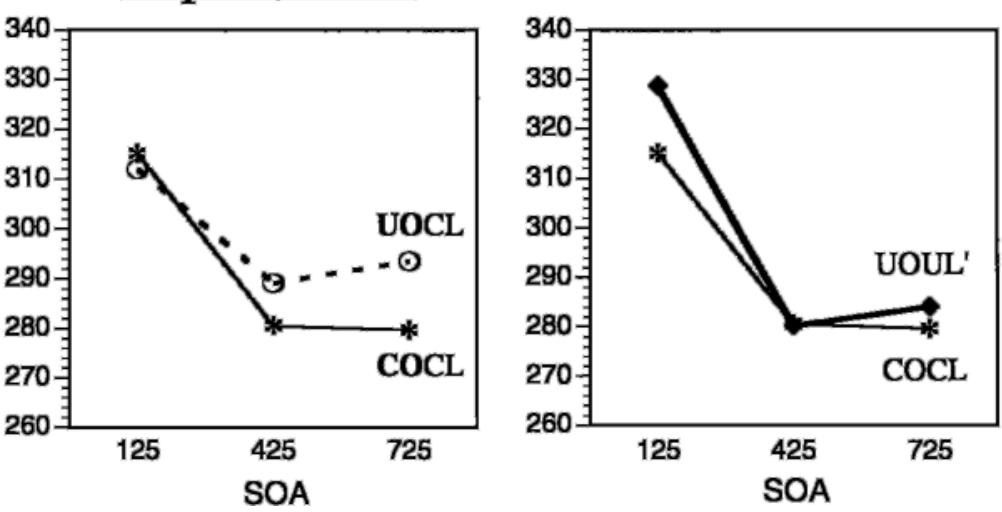

Figure 2. Results from Experiments 1 and 2 (top vs. bottom rows, respectively). Left panels: Mean RT as a function of location cuing ( $U L=$ uncued location, $C L=$ cued location) when objects were always uncued shapes. Center panels: Effect of object cuing (UO = uncued object, $\mathrm{CO}=$ cued object) for targets appearing in cued locations. Right panels: Effect of location-plus-object cuing when there was one cued and one uncued shape in the display.

msec SOA $[F(1,22)=22.9, p<.001]$. Thus, significant object-based facilitation was observed at the same SOA as location-based inhibition.

Location-plus-object cuing. For contrast, we separately analyzed trials in which only the uncued marker changed shape (COCL and UOUL'). Object and location cuing are confounded in this comparison, making it an analysis of location-plus-object ( $\mathrm{Loc}+\mathrm{Obj}$ ) cuing. However, this provided an analysis of Loc + Obj cuing that could be compared with the previous analyses. The factors in this analysis were Loc + Obj cuing and SOA. Results showed a main effect of Loc + Obj cuing, indicating that target detection was faster overall for cued than for uncued targets $[F(1,11)=6.78, p<.05]$. Interestingly, there was no interaction with SOA $[F(2,22)=2.96$, $p>$.05]. Consistent with the effects of location cuing, simple comparisons revealed a significant benefit for cued locations at the short SOA $[F(1,22)=15.54, p<$
$.001]$. In contrast, no cuing effects were observed at the longer SOAs $[F(1,22)=1.78$ and $F<1$, respectively]. At the long SOAs, neither location-based IOR nor the advantage for cued objects was observed to dominate (Figure 2, top right).

\section{Accuracy}

Table 1 shows the mean RTs for correct responses and the miss and false alarm rates for Experiment 1. Overall, performance was very good. The participants accurately detected $97.9 \%$ of the targets and missed $1.3 \%$. The remaining $0.8 \%$ were detected also but were excluded from analysis because of eye movements. On trials in which no target was presented, the participants made false alarms $1.4 \%$ of the time. As can be seen in the table, miss rates for the various SOAs were similar regardless of whether one or both location markers changed in shape. There were slightly more false alarms, however, when only one 
Table 1

Mean Response Times (RTs), Miss Rate, and False Alarm Rate as a Function of

Location Marker Status and Cue-to-Target Interval

Cue-to-Target Interval (msec)

\begin{tabular}{|c|c|c|c|c|}
\hline \multirow[b]{2}{*}{ Location Marker Status } & & & & \multirow[b]{2}{*}{ False Alarms } \\
\hline & 125 & 425 & 725 & \\
\hline \multicolumn{5}{|l|}{ Change in both markers } \\
\hline Mean RT & 430 & 403 & 415 & \multirow[t]{2}{*}{$0.2 \%$} \\
\hline Misses (\%) & 0.11 & 0.26 & 0.35 & \\
\hline \multicolumn{5}{|l|}{ Change in uncued marker } \\
\hline Mean RT & 430 & 399 & 406 & \multirow[t]{2}{*}{$1.2 \%$} \\
\hline Misses (\%) & 0.04 & 0.26 & 0.30 & \\
\hline
\end{tabular}

Note-total misses $=1.3 \%$; total false alarms $=1.4 \%$.

location marker changed. The total number of false alarms was very small, so no formal analyses were conducted.

\section{Discussion}

Our aim in Experiment 1 was to separate location and object cuing effects while keeping offsets balanced on the two sides of the display. When both location markers changed in shape, location cuing effects paralleled those found in earlier experiments with unchanged location markers: Facilitation of the cued location was observed at the short SOA, and inhibition of the cued location was observed at the long SOA (left panel, Figure 2). Thus, the biphasic pattern associated with exogenous spatial cuing was unaffected by uniform changes in the shapes of the location markers after cuing. It would seem that location-marker identity has little or no influence on mechanisms of reflexive attentional orienting to spatial locations.

However, a different pattern emerged on trials in which the uncued location marker changed in shape and the cued location one did not (right panel, Figure 2), producing two differently shaped location markers in the final display. At the short SOA, responses still showed the expected facilitation at the cued location. In contrast, the expected inhibition of the cued location at longer SOAs was no longer observed: Instead, RTs were equally fast to cued and uncued locations (COCL and UOUL'). IOR was apparently eliminated when a unilateral shape change resulted in two differently shaped location markers in the target display. Although facilitation of the cued location survived shape changes as well as shape differences in the display, IOR did not. At this point, the reason for the lack of IOR is unclear. It is possible that either the unilateral shape change or shape differences in the display disrupted exogenous inhibitory processes. A theoretically more interesting possibility, however, is suggested by the analysis of object cuing.

The analysis of object cuing (at cued locations) revealed a late-onset advantage for cued objects. Unfortunately, because this analysis compared the UOCL targets from trials in which both shapes had changed and the COCL targets from trials in which only one shape had changed, it is unclear whether the observed cued object advantage represents a type of object-based facilitation or the disruption of location-based IOR in displays con- taining a unilateral shape change. Reductions in locationbased IOR following unilateral shape changes would have likewise benefited the COCL condition. Notwithstanding, to the extent that IOR is not eliminated by unilateral shape changes or shape differences in the display, an intriguing possibility is that object-based facilitation may compete with location-based inhibition at long SOAs. Unfortunately, Experiment 1 did not include all the possible combinations of object and location cuing, so the interaction between the two could not be tested. Experiment 2 tested this interaction directly and further examined the effects of unilateral shape changes and shape differences on exogenous spatial orienting.

\section{EXPERIMENT 2}

In order to include a condition in which the cued object appeared in an uncued location while bilateral offsets were maintained, we added two flanking horizontal bars to the Star of David stimuli, one above and one below each star (Figure 3). These bars always disappeared with the removal of the cue, resulting in a bilateral visual offset on every trial. The Stars of David either did not change at all, changed bilaterally in a uniform fashion (similar to the both change trials in Experiment 1), changed bilaterally into two different shapes, or changed unilaterally at either the cued location or the uncued location. The inclusion of both types of unilateral change trials in this experiment allowed us to test all combinations of cued and uncued objects and locations and to examine the interaction between object and location cuing.

The no-change trials allowed us to confirm that typical location cuing effects were still observed despite the disappearance of the flanking lines on both sides of the display and the presence of shape changes on other trials.

The bilateral change condition was included to test for differences owing to shape mismatch in the display. In the bilateral uniform change condition, both stars changed to the same shape, either diamonds or rectangles. These trials were equivalent to those in Experiment 1, in which both markers changed into triangles, except that different shapes were used for the uncued objects. In the bilateral different change condition, each star changed to a different shape (one diamond and one rectangle). Thus, there were always two uncued objects (same or different) in the target display, allowing us to test how shape differences, in the absence of a cued object, affected the pattern of location-based orienting.

Finally, in order to make sure that the results of Experiment 1 were not due to the specific shapes tested, we used different segments of the initial Star of David stimulinamely, diamonds and indented rectangles. In the previous experiment, the Star of David stimuli could be easily parsed into their two component triangles. Although the diamonds and indented rectangles used here were also components of the Star of David, they were not as easily perceived as such. When asked, the participants reported that they were unaware that the shapes were linked in any way. Thus, a replication of the previous results with these 
alternate shapes would strengthen our account that the uncued objects were perceived as new shapes, rather than as preexisting parts of the initial star stimuli.

\section{Method}

\section{Participants}

Nineteen (11 female, 8 male) University of California, Davis undergraduates were recruited. All were naive as to the purpose of the study. Fourteen received psychology course credit for their participation, whereas the rest were compensated $\$ 10 / \mathrm{h}$ for their time. All of the participants reported normal or corrected-to-normal vision, and 1 participant was left-handed. The data from 3 participants were excluded because there were indications that they had not followed instructions. One was excluded because $20 \%$ of her trials were accompanied by eye movements. The other two were excluded on the basis of excessive false alarm rates $(34 \%$ and $9 \%$, respectively), indicating a strong bias to respond whether the target had appeared or not. Data from the remaining 16 participants ( 8 female, 8 male) are reported below.

\section{Stimuli and Apparatus}

Stimulus displays were the same as those in Experiment 1, except that each Star of David stimulus was initially presented with two horizontal flanking lines, one above and one below each star. The horizontal bars were $2.1 \mathrm{~cm}$ long and were placed $0.1 \mathrm{~cm}$ above and below the stars. These bars were present until the moment the cue was removed. Stimulus displays following the cue could consist of any combination of Star of David stimuli, diamonds, or indented rectangles. Diamonds subtended $2.5^{\circ} \times 1.4^{\circ}$ of visual angle and were created from the Star of David stimuli by offsetting all lines except those delineating the central diamond shape. The indented rectangles were similarly created from the Star of David stimuli and subtended $1.1^{\circ} \times 2.1^{\circ}$ of visual angle. Targets (\#) were presented in bold Helvetica font size 34 and appeared with equal likelihood in the center of one of the remaining shapes (diamond, rectangle, or star).

\section{Procedure}

The procedures and initial stimulus displays were identical to those used in Experiment 1, except for the addition of the horizontal flanking lines above and below each Star of David. Eye movements were monitored as described previously, and trials with eye movements were marked by keypress and excluded from further analysis. Cuing was implemented as before, but when the cue offset, the horizontal lines flanking each location marker also disappeared. Cues were not predictive of target location, and the same cue-totarget SOAs and target and stimulus durations were used as those in Experiment 1. Location marker changes were achieved as described previously via partial offset of the Star of David at the time of cue offset. The resulting shapes could be diamonds or indented rectangles. The following types of location marker changes were randomly presented, with the constraint that no more than three trials in a row would consist of the same type of location marker change.

No change. On $25 \%$ of the trials (192 target trials, 16 catch trials), there was no change in the location markers, except for the bilateral offset of the flanking lines. On these trials, the location markers began and ended as stars. Therefore, all targets in the nochange trials appeared within cued objects. Half of the targets appeared at the cued location (COCL), and the other half appeared at the uncued location (COUL; see Figure 3).

Bilateral uniform change. On $25 \%$ of the trials (192 target trials, 20 catch trials), both location markers changed shape in uniform fashion. On these trials, the location markers began as stars and then changed into two diamonds or two rectangles with equal likelihood. Thus, all the targets in the bilateral uniform trials appeared in uncued objects. Half of the targets appeared at the cued location (UOCL), and the other half appeared at the uncued location (UOUL).
Bilateral different change. Another $25 \%$ of the trials (192 target trials, 20 catch trials) contained a bilateral shape change that resulted in two different uncued objects. One star changed to a diamond, and the other to an indented rectangle. Each shape/location was equally likely to contain the target, resulting in equal numbers of UOCL and UOUL targets.

Unilateral change. The remaining 25\% of the trials (192 target trials, 20 catch trials) contained a unilateral shape change at either the cued location or the uncued location. One star remained unchanged, whereas the other changed into either a diamond or a rectangle. Therefore, targets appeared with equal likelihood in a cued object at the cued location (COCL condition), a cued object at the uncued location (COUL condition), an uncued object at the cued location (UOCL condition), or an uncued object at the uncued location (UOUL' condition).

Nine percent of the trials were catch trials. Each experimental session consisted of 76 catch trials, 384 cued targets, and 384 uncued targets, for a total of 844 trials. The participants were given a short break after every 85 trials and a longer break to stretch and walk around at the halfway point. Location cuing was pseudorandomly varied so that no more than 3 trials in a row contained a target of the same location cuing status. SOA and target visual field were also pseudorandomly varied, with no more than 4 trials in a row sharing the same SOA or target visual field, respectively. Similar to Experiment 1, far more targets appeared in uncued objects than in cued objects (62\% and $38 \%$, respectively), which, if anything, should have biased the participants against cued objects.

\section{Results}

\section{Response Time}

Several planned analyses were carried out on specific subsets of the data, which allowed for different hypotheses to be tested. All the analyses were conducted on trimmed means, using the same criteria as those described in Experiment 1 .

Replication. The first analysis was conducted to determine whether the location and object cuing effects observed in Experiment 1 were replicated. These analyses were performed on the bilateral uniform change trials (UOCL and UOUL) and on trials containing a unilateral change at the uncued location (COCL and UOUL'), because these trials were equivalent to those tested in Experiment 1, with the exception of the shapes used for uncued objects (compare Figures 1 and 3 ) and the addition of the flanking bars. The replication data are plotted in the lower portion of Figure 2.

To test for replication, these trials were entered into an ANOVA with those from Experiment 1. Since the participants in each experiment were chosen from essentially the same population, experiment ( 1 vs. 2 ) was analyzed as a between-subjects factor, whereas cuing condition (UOCL, UOUL, UOCL, or COCL) and SOA (125, 425, or $725 \mathrm{msec}$ ) were within-subjects factors. There was a significant difference in detection times between the two experiments $[F(1,26)=62.4, p<.001]$, with faster detection times in Experiment $2(M=297 \mathrm{msec})$ than in Experiment $1(M=414 \mathrm{msec})$. This difference, however, was strictly additive and did not interact with cuing condition or SOA (both $F_{\mathrm{S}}<1.9$ ). The three-way interaction also was nonsignificant $(F<1)$. Thus, neither the addition of the flanking lines above and below each location marker nor the different shapes altered the cuing patterns previ- 


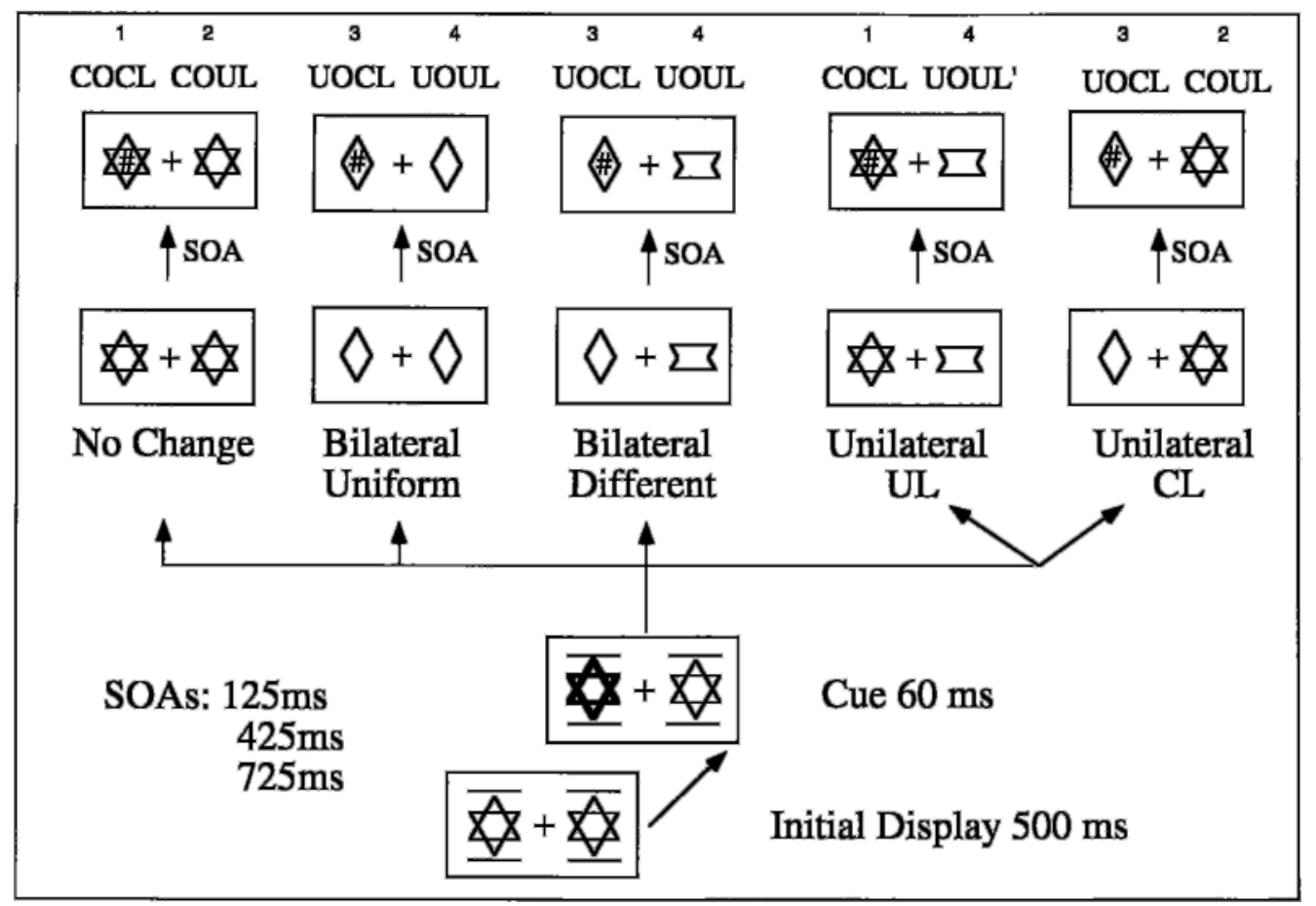

Figure 3. Stimulus sequences for Experiment 2: Flanking lines presented in the initial stimulus display always disappeared simultaneously with the cue, ensuring bilateral visual offsets on all trials. There were four different location marker conditions. There was no change in the location markers on $25 \%$ of the trials. Bilateral shape changes consisted of both shapes changing in the same way (bilateral uniform, $25 \%$ of the trials) or into two different shapes (bilateral different, $25 \%$ of the trials). The remaining trials contained a unilateral shape change, which was equally likely to occur at the uncued location (UL) or the cued location (CL). The target is illustrated in the cued location for each condition but was equally likely to occur in either the CL or the UL. The labels at the top of the diagram indicate all the resulting trial types. Each label first specifies whether the target would be presented in a cued or an uncued object (CO or UO) and then indicates whether it is in a CL or a UL. The number above each label corresponds to one of the four possible combinations and, where the numbers are similar, they indicate identical trial types within the various location marker conditions.

ously observed. Comparison of the UOUL and UOUL' conditions again revealed virtually identical RTs across SOAs, as was observed in Experiment $1\left(M_{\mathrm{UOUL}}=326\right.$, 283, and $287 \mathrm{msec}$ for 125-, 425-, and 725-msec SOAs, respectively; $M_{\mathrm{UOUL}}=329,280$, and $284 \mathrm{msec}$, respectively), indicating that offsets on the opposing side of the display were equivalent whether they consisted of the removal of the cue, flanking bars, and partial object (UOUL) or just the cue and flanking bars (UOUL').

No change and bilateral shape changes. Object cuing was held constant within each of these conditions, so analyses of location cuing were conducted in order to determine whether extraneous factors might be responsible for altering the typical pattern of location-based orienting. The data from each type of location marker change are plotted in Figure 4 to reflect the effects of location cuing.

The no-change trials (Figure 4A) were traditional Posner cuing trials, except for the Star of David shapes and flanking lines that were offset bilaterally on each trial. One concern was how the removal of the flanking lines might affect orienting. A second concern was whether shape changes on other trials within the session could have altered the participant's attentional set, thereby altering the typical pattern of reflexive orienting. A 2 (location cuing $) \times 3(\mathrm{SOA})$ ANOVA revealed a significant two-way interaction between location cuing and SOA $[F(2,30)=$ $31.58, p<.001]$, indicating significant facilitation of cued locations at the $125-\mathrm{msec}$ SOA $[F(1,30)=30.47$, $p<.001]$ and significant IOR at $725 \mathrm{msec}[F(1,30)=$ $32.03, p<.001]$. A main effect of SOA $[F(2,30)=$ $32.05, p<.001]$ demonstrated that RTs generally decreased with longer SOAs. Thus, typical exogenous spatial cuing effects were observed for the trials without shape changes, even though these trials contained bilateral offsets and were intermingled with trials that did contain shape changes.

In the bilateral change condition, shape changes resulted in either two identical or two different uncued shapes in the target display (see Figure 3). Comparing these two trial types allowed us to investigate the effect of shape differences on location cuing. If location cuing were unaffected by shape differences in the display, equivalent levels of facilitation and IOR should be observed 

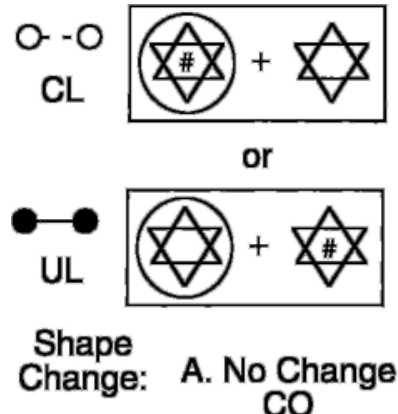

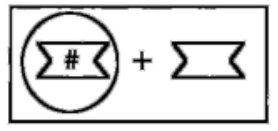

or

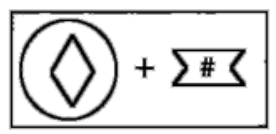

B. Bilateral UO

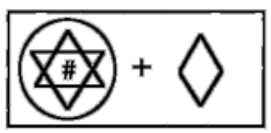

or

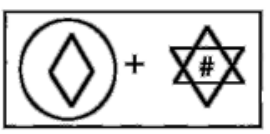

C. Unilateral CO
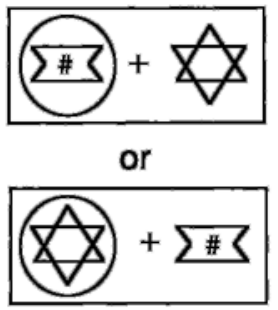

D. Unilateral UO

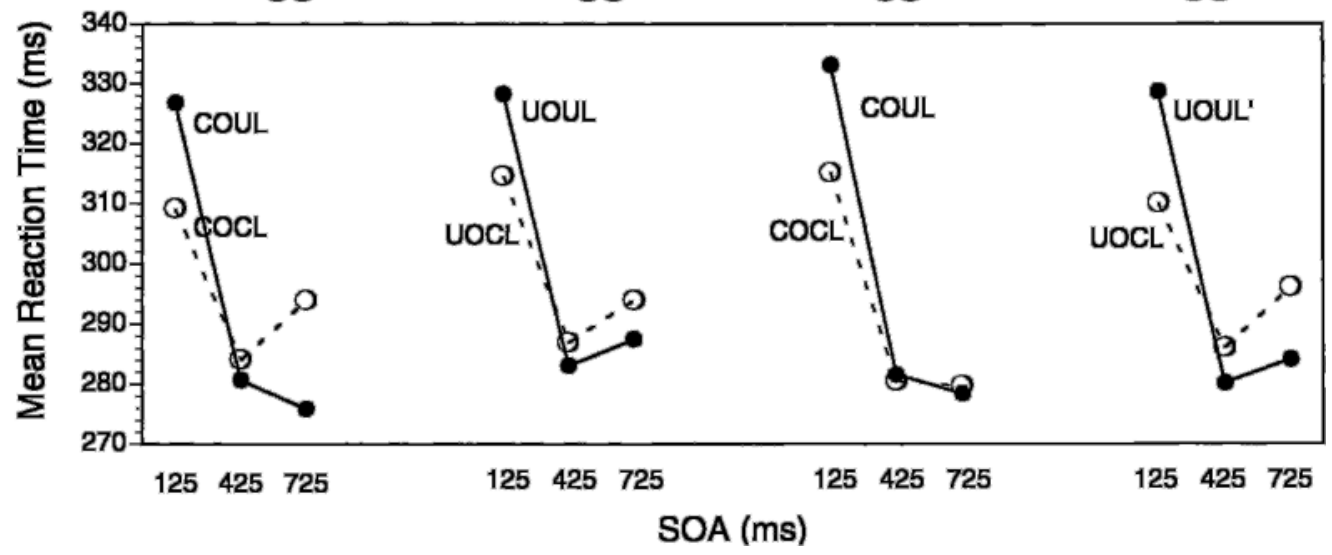

Figure 4. Location cuing effects and schematic displays for each location marker condition (type of shape change) in Experiment 2. The circled shape indicates the cued location; thus, targets illustrated in the top row are presented in cued locations (CLs), and those depicted in the lower row are presented in uncued locations (ULs). Each graph (A-D) depicts a comparison between the CLs and the ULs, as depicted in the schematic above. Object cuing is held constant for each comparison but varies with type of shape change. (A) No change in shape resulted in two cued objects (COUL vs. COCL). (B) Bilateral shape changes (uniform and different combined) resulted in two uncued objects (UOUL vs. UOCL). Unilateral shape changes resulted in both (C) cued objects (COUL vs. COCL) and (D) uncued objects (UOUL' vs. UOCL).

whether there were two identical or two different shapes in the display. Such a result would indicate that the attenuation of IOR observed previously was not due to shape differences per se but, perhaps, to the competitive influence of a cued object. A $2 \times 2 \times 3$ ANOVA on the bilateral change trials in which shape difference (identical or different), location cuing (cued or uncued), and SOA $(125,425$, or $725 \mathrm{msec})$ were within-subjects factors, revealed no effects of shape difference $(F<1)$. Mean RTs were equally fast for displays containing two different or two identical shapes. The expected location cuing $\times$ SOA interaction was significant $[F(2,30)=10.91, p<$ $.001]$, but shape difference did not interact with either variable. Nor did shape difference interact with location cuing when the analysis was restricted to the long SOA $(F<0.01)$, indicating similar levels of IOR regardless of shape mismatch. These results are consistent with the idea that the cued object advantage observed above was due to object-specific facilitation, which may indeed compete with location-based IOR.

Unilateral shape changes. Unlike Experiment 1, all combinations of cued locations/uncued locations and cued objects/uncued objects (COCL, UOCL, UOUL', and COUL) could be examined within the unilateral change trials. Thus, we subjected these trials to an ANOVA, with location cuing (cued location or uncued location), object cuing (cued object or uncued object), and SOA (125, 425 , or $725 \mathrm{msec}$ ) as within-subjects factors. RT decreased over SOA $[F(2,30)=45.33, p<.001]$, replicating previous observations.

A significant location cuing $\times$ SOA interaction $[F(2,30)=17.87, p<.001]$ revealed the expected pattern of facilitation at the shortest SOA $[F(1,30)=33.62$, $p<.001]$ and inhibition at the longest SOA $[F(1,30)=$ $4.42, p<.05]$. In contrast, a significant object cuing $\times$ SOA interaction $[F(2,30)=6.09, p<.01]$ revealed no effect of object cuing at the early SOAs, followed by a facilitatory effect at the longest SOA. At $725 \mathrm{msec}$, cued object targets were more rapidly detected than uncued object targets $[F(1,30)=11.82, p<.01]$. There was also a significant interaction between object and location cuing $[F(1,15)=4.80, p<.05]$, reflecting a larger object cuing effect at the cued location. The difference between cued and uncued objects was significant at the cued location $[F(1,15)=9.44, p<.01]$, but not at the uncued location.

Importantly, unilateral shape changes did not obscure location-based IOR within uncued objects, eliminating this account of the cued object advantage at the cued lo- 


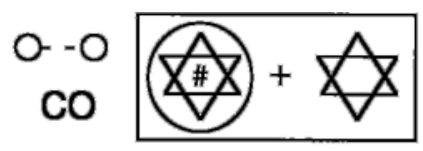

or
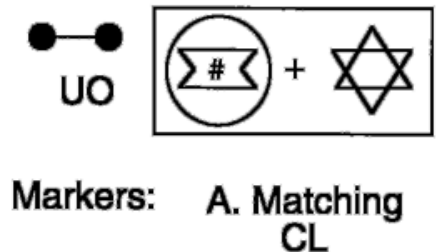

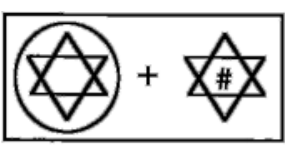

or

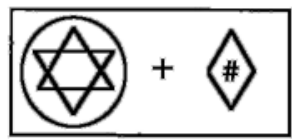

B. Matching UL

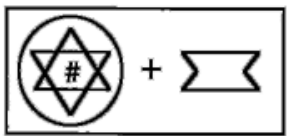

or

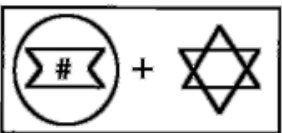

C. Nonmatching $\mathrm{CL}$

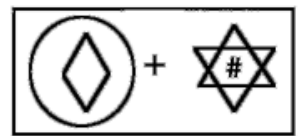

or

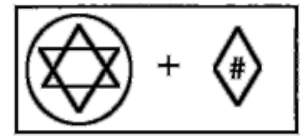

D. Nonmatching UL

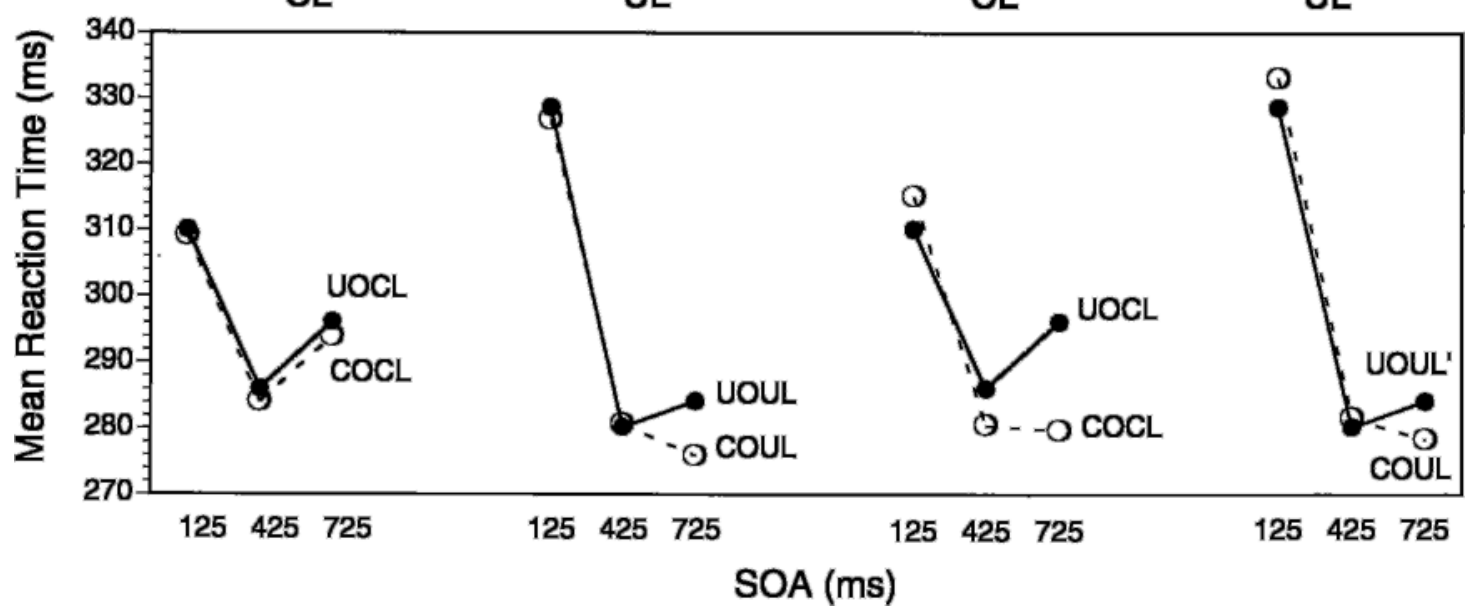

Figure 5. Experiment 2: Data from the previously shown no-change and unilateral change trials are replotted here to reflect the effects of object cuing. Schematics again illustrate the two trial types being compared in the graph below: Circles represent the cued location, whereas stars represent cued objects $(\mathrm{CO})$, and all the other shapes represent uncued objects (UO). Each plot (A-D) depicts a comparison between $\mathrm{CO}$ and $\mathrm{UO}$ targets, while location cuing is held constant. Panels A and B reflect comparisons between the no-change CO trials (top schematic), which contained two matching shapes in the target display, and the unilateral UO trials (bottom schematic). Panels C and D show object cuing effects for the unilateral change condition, which always resulted in two nonmatching shapes in the target display.

cation. Thus, it seems that a late onset advantage for cued object may compete with location-based IOR at cued locations. The three-way object cuing $\times$ location cuing $\times$ SOA interaction, however, did not reach significance. This is probably because competition between objects and locations occurred in only one (COCL) of the four cuing conditions, and then only at the longest SOA.

As in Experiment 1, planned comparisons were carried out to further isolate the effects of object cuing from location cuing. In contrast to Experiment 1 , however, Experiment 2 was designed so that the effects of location cuing could be examined for targets appearing in either cued objects or uncued objects (Figures 4C and 4D) and the effects of object cuing could be examined at either the cued location or the uncued location (Figures 5C and 5D).

Location cuing. In Figures 4C and 4D, the data from the unilateral change trials are grouped according to whether the target appeared in a cued object or an uncued object. If location cuing were blind to the type of objects present, the same biphasic pattern of location cuing (early facilitation, late IOR) should be obtained for both cued object and uncued object alike. This was not the case. As is shown in Figure 4, the location cuing effects for unilateral uncued object targets (Figure 4D) resembled the effects observed for the no-change and bilateral change trials and replicated the results of Experiment 1. There was significant facilitation of cued locations (UOCL) at the short SOA $[F(1,30)=12.28, p<.01]$ and significant IOR at the longest SOA $[F(1,30)=5.11, p<.05]$. In contrast, for the unilateral cued object targets (Figure 4C), there was significant facilitation of the cued location at the short SOA $[F(1,30)=11.55, p<.01]$, as was expected, but no indication of location-based IOR.

Object cuing. Data were replotted in Figure 5 to reveal the effects of object cuing. Panels $C$ and $D$ show the effects of object cuing for the unilateral shape change condition, in which, incidentally, there were always two nonmatching shapes in the final display. Object cuing was separately examined at cued and uncued locations. If cued objects were facilitated at long SOAs independently of their location, detection of cued object targets should be faster than that for uncued object targets whether the target's location had been cued or not. This was not the case. For targets appearing in the cued loca- 
Table 2

Mean Response Times (RTs) and

Miss Rates for Experiment 2 as a Function of Location Marker Change and Cue-to-Target Interval

\begin{tabular}{llll}
\hline & \multicolumn{3}{c}{ Cue-to-Target Interval $(\mathrm{msec})$} \\
\cline { 2 - 4 } Location Marker Change & 125 & 425 & 725 \\
\hline No change & 318 & 283 & 286 \\
$\quad$ Mean RT & 0.05 & 0.30 & 0.28 \\
$\quad$ Misses (\%) & & & \\
Bilateral uniform change & 320 & 286 & 290 \\
$\quad$ Mean RT & 0.13 & 0.38 & 0.31 \\
$\quad$ Misses (\%) & & & \\
Bilateral different change & 324 & 285 & 291 \\
$\quad$ Mean RT & 0.11 & 0.27 & 0.36 \\
$\quad$ Misses (\%) & & & \\
$\quad$ Unilateral change & 322 & 282 & 285 \\
$\quad$ Mean RT & 0.11 & 0.29 & 0.35 \\
$\quad$ Misses (\%) & &
\end{tabular}

Note-Total misses $=3 \%$; total false alarms $=1.6 \%$.

tion (Figure 5C), the findings of Experiment 1 were replicated, demonstrating a significant advantage for cued objects at $725 \mathrm{msec}[F(1,30)=9.58, p<.01]$. However, for targets appearing in uncued locations (Figure 5D), there was no effect of object cuing at any SOA $(F<1, F<1$, and $F=1.16$ for $125-, 425-$, and 725 msec SOAs, respectively). Object cuing effects for trials with matching shapes (Figures 5A and 5B) are discussed next.

Object cuing with matching shapes. In order to determine how robust the cued object advantage was, we conducted the same analysis as described above for the unilateral change trials, but replaced the unilateral COCL and COUL means with the corresponding means from the no-change trials (Figures 5A and 5B). Thus, the same four cells were analyzed (COCL, COUL, UOUL', and UOCL), but in this case, the cued object trials came from displays with matching shapes. Although shape mismatch was not observed to alter location cuing, it may have played a role in eliciting object-based orienting. Note also in these comparisons that the status (changed or unchanged) of the object on the opposite side of the display was held constant.

The 2 (location cuing) $\times 2$ (object cuing) $\times 3$ (SOA) ANOVA revealed a main effect of SOA $[F(2,30)=35.1$, $p<.001]$ and a highly reliable interaction between location cuing and SOA $[F(2,30)=22.8, p<.001]$. In contrast to the previous analysis, however, there were no significant effects involving object cuing when there were matching shapes in the display (all $F_{\mathrm{S}}<1.6$ ). These data suggest that the cued object advantage observed above may be produced only in displays that contain mismatching shapes. This implicates shape mismatch as an important factor in eliciting object-based orienting. Before we can make such a conclusion, however, we must determine whether it is the shape mismatch alone or the presence of a within-trial shape change that is most influential.

\section{Accuracy}

Table 2 shows the mean RTs for correct responses and the miss rates for Experiment 2. Performance was very accurate. The participants accurately detected $97 \%$ of the targets. Fewer than $1 \%$ of the trials were excluded for eye movements or anticipatory responses. The false alarm rate on trials in which no target was presented was $1.6 \%$. As can be seen in the table, at each SOA, similar miss rates and mean RTs were observed across the various location marker conditions.

\section{Discussion}

The effects of location cuing replicated earlier studies of exogenous spatial orienting. cued locations were facilitated at short SOAs and inhibited at long ones. A new finding is that shape changes have no effect on the facilitatory component of exogenous orienting (cf. Figures 4A-4D). Consistent levels of facilitation were obtained in each location marker condition, suggesting that at early SOAs, attention is reflexively allocated to cued locations independently of the objects that occupy those locations. In contrast, the inhibitory component of exogenous orienting was affected by location marker status. In displays containing mismatching shapes, IOR appeared to be attenuated when a cued object occupied the cued location (Figure 4C). However, when there was no cued object present, IOR was unaffected by shape mismatch in the display.

The effects of object cuing suggest that, as in the cued location conditions of Experiment 1, cued objects are facilitated at long SOAs, particularly in displays containing nonmatching shapes. The most plausible account is that shape differences in the target display drew attention to the shape dimension, making object facilitation possible. When cued objects cannot be differentiated on the basis of their shape (as in the matching-shapes trials), only the effects of location cuing are observed. Following a unilateral shape change, however, the cued object can be distinguished from the uncued object on the basis of shape, and under these circumstances an advantage for cued objects is observed at the long SOA. This attentional capture by the cued object, however, produces no apparent cost to the uncued object presented with it, as might be expected from space-based models of attention. UOUL' targets were presented opposite a cued object, whereas UOULs were not, yet they resulted in identical RTs.

The nonmatching COCL condition is the one condition in which both location and object cuing effects are expected to be present but to affect RTs in opposite directions. Location-based IOR should slow detection at the long SOA, whereas object facilitation should hasten it. If the two effects compete, the COCL condition should result in latencies that are intermediate between the COUL condition (which should be fast at the long SOA, owing to the lack of location-based inhibition and possible cued object advantage) and the UOCL condition (which 
should be slow, owing to unchecked inhibition of the cued location). This pattern was present. Thus, the lateonset advantage observed for cued objects under conditions of mismatching shapes seems to compete with location-based IOR at the cued location. Our hypothesis is that when both object and location are cued, the outcome of the conflict between location-based IOR and object facilitation at the long SOA depends on attention being attracted to the shape dimension. If there is a single shape change, resulting in two different shapes in the display, object-based facilitation determines RT by canceling location-based IOR. On the other hand, if neither or both shapes change, the participant is confronted with either two indistinguishable shapes or a display that lacks a cued object altogether. Under these conditions, locationbased attentional orienting dominates.

At this point, we cannot conclude whether it is the presence of a uniquely shaped cued object, or the presence of a shape change that is responsible for eliciting the observed object effect. Thus far, all the trials containing a shape mismatch have also included a shape change. Experiment 3 was designed to examine this issue. Although it is possible that shape mismatch is the critical factor for eliciting object-based orienting, it is equally possible that the significance of a shape difference in the display depends on the presence of a preceding shape change within the trial. Evidence from both Experiment 2 (no differences between the bilateral uniform and the bilateral different trials) and Experiment 3, still to be described, suggests that the crucial factor is not the shape difference itself, but the presence of a shape change.

Note, however, that unilateral shape changes did not result in attentional capture. If unilateral shape changes had captured attention, the opposite pattern would have been obtained for object cuing; detection would be faster for targets appearing in changed (uncued) objects. Moreover, identical RTs were observed for the UOUL' and the UOUL conditions, which contained unilateral and bilateral shape changes, respectively. This lack of a capture effect is not so surprising, given that there were bilateral (and simultaneous) visual offsets on every trial.

\section{EXPERIMENT 3}

The results from Experiment 2 suggest that displays containing two different shapes (one of which was cued) elicit object-based orienting, whereas displays containing identical shapes do not. Yet, it is still unclear whether the cued object advantage at the long SOA is contingent on the presence of different shapes in the display or on the occurrence of a shape change within the trial. Experiment 3 explored the effects of shape mismatch in the absence of any within-trial shape changes.

In this experiment, the location markers varied only between trials and consisted of either two identical (matching) shapes or two different (nonmatching) shapes that remained present throughout the trial. If shape mismatch per se is critical for generating faster RTs to cued objects at long SOAs, detection times for targets appearing in cued location markers should be faster at long SOAs in displays with nonmatching shapes, as compared with displays with matching shapes. Moreover, an advantage for cued object targets at the long SOA should compete with any location-based IOR accruing in response to the exogenous precue. Therefore, IOR should be absent or reduced in displays containing nonmatching versus matching shapes.

\section{Method}

\section{Participants}

Ten ( 5 female, 5 male) University of California, Davis undergraduates, all naive as to the experimental paradigm, received course credit for their participation in the study. All reported normal or corrected-to-normal vision, and all but one were righthanded.

\section{Stimuli and Apparatus}

The same stimuli, apparatus, and eye-monitoring technique were used as in Experiment 2, except that there were no within-trial shape changes and no flanking lines in the display.

\section{Procedure}

The procedures were identical to those in the no-change condition of Experiment 2. The initial stimulus display consisted of the central fixation cross and two location markers, which could be two diamonds, two indented rectangles, or one of each shape. Half of the trials contained two location markers of the same shape (matching), and half contained two different shapes (nonmatching). As before, the cue was nonpredictive and consisted of thickening one of the two location marker shapes for $60 \mathrm{msec}$. For simplicity, the cueto-target SOA was either 125 or $725 \mathrm{msec}$.

The participants were told that neither the cues nor the location marker shapes were predictive of the upcoming target and that targets were equally likely to appear on either side of fixation. Both speed and accuracy of response were emphasized. Each participant completed a practice run of 20 trials prior to completing the experimental session, which included 40 catch trials ( $9 \%$ of the total), 192 cued targets, and 192 uncued targets. A short break was permitted after 71 successive trials.

The variables were cuing (cued or uncued), shape mismatch (matching or nonmatching), and SOA (125 or $725 \mathrm{msec}$ ), resulting in a total of eight experimental conditions. Equal numbers of targets were assigned to each condition and then presented in random order. Target visual field and location marker shape (diamond or rectangle) were counterbalanced across experimental conditions but were otherwise presented randomly.

\section{Results}

\section{Response Time}

Analyses were conducted on trimmed means, using the same criteria as described in Experiments 1 and 2. The data were submitted to a 2 (shape mismatch) $\times 2$ (cuing) $\times 2$ (SOA) ANOVA, which revealed a significant main effect of SOA $[F(1,9)=5.63, p<.05]$ that replicated the previous findings and a significant cuing $\times$ SOA interaction $[F(1,9)=27.95, p<.001]$. An analysis of the cuing $\times$ SOA interaction demonstrated a pattern typical of exogenous spatial orienting: significant facilitation at the short SOA $[F(1,9)=6.16, p<.05]$, followed by significant IOR at the long SOA $[F(1,9)=24.95, p<.001]$. Importantly, there were no significant effects involving 

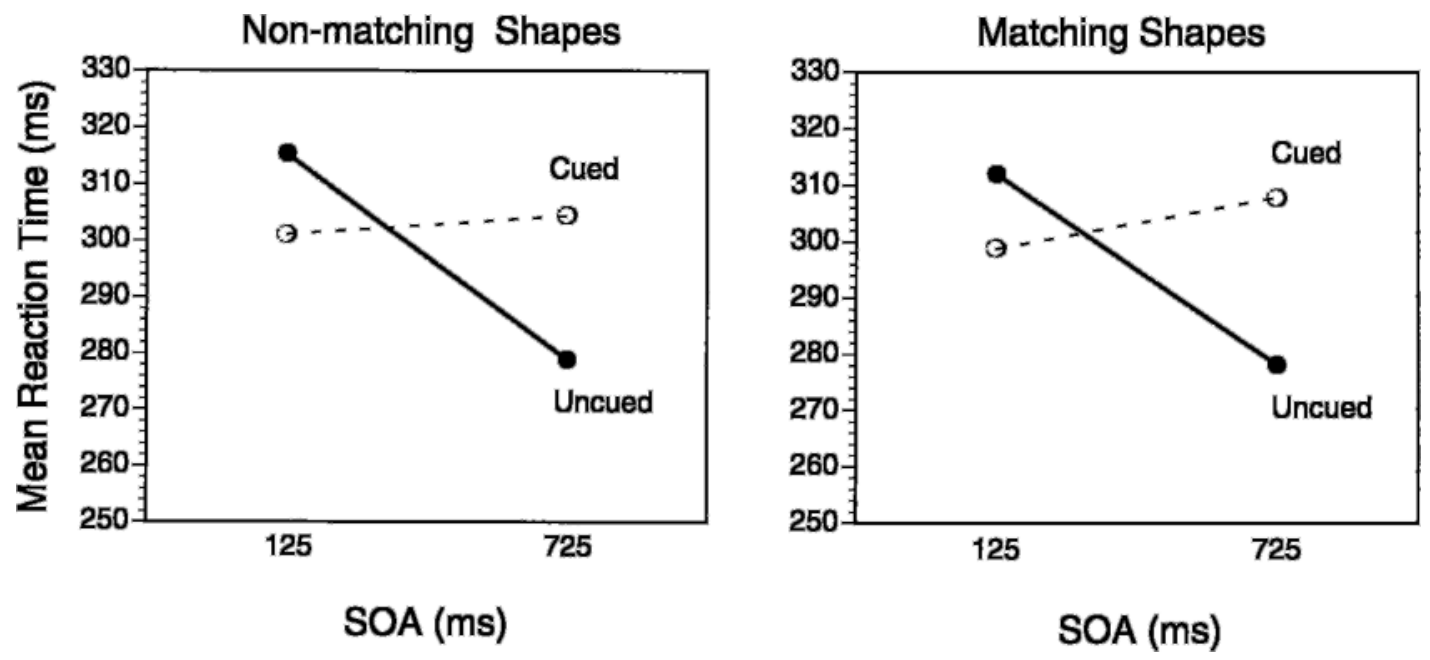

Figure 6. Experiment 3: Comparison of cuing effects in displays containing two different (nonmatching) or two matching shapes from the beginning to the end of the trial. Shape mismatch in static displays did not evoke objectbased orienting at the long SOA.

shape mismatch (Figure 6). Similar RTs and equivalent magnitudes of IOR were observed whether the location markers matched in shape or not, indicating that typical location cuing effects dominated in these static displays. Finally, a planned comparison at the long SOA confirmed that shape mismatch, in the absence of shape changes, had no significant effect on RTs to targets appearing in cued objects $[F(1,9)=2.38, p>.10]$.

\section{Accuracy}

Target detection performance was very good. The participants accurately detected $98 \%$ of the targets and missed $1 \%$. The remaining $1 \%$ were excluded for anticipatory responses $(0.7 \%)$ and/or eye movements $(0.4 \%)$. The false alarm rate on trials in which no target was presented was $0.5 \%$.

\section{Discussion}

In this experiment, either two matching or two nonmatching shapes were presented at stimulus onset. Since there were no shape changes during the trial, a cued object was always present in the target display. The results showed that the presence of mismatching shapes alone was not sufficient for eliciting object-based orienting or the corresponding attenuation of IOR. Instead, shape changes within the trial (as in Experiments 1 and 2), rather than static differences in shape, appear to be a critical factor in influencing the reflexive allocation of attention. The attenuated IOR observed in Experiments 1 and 2 thus seems to be a consequence of a shape change that served to distinguish the cued object from the uncued object in the display. We could rule out the possibility that IOR was attenuated by any unilateral shape change, because significant IOR was observed following a unilateral shape change in Experiment 2 (Figure 4D).

\section{EXPERIMENT 4}

Experiments 1 and 2 always used stars as the initial stimuli, followed by stars or components of the stars in the final target display. The effect of object cuing could have been confounded with differences in the visibility of the target (\#) within the stars, as compared with the other shapes. Experiment 4 was designed to compare target detection within stars, diamonds, and indented rectangles under identical cuing conditions.

\section{Method}

\section{Participants}

Nine (4 female, 5 male) University of California, Davis undergraduates received course credit for their participation in the study. None had participated in the previous experiments. All of the participants reported normal or corrected-to-normal vision, and all 9 were right-handed.

\section{Stimuli and Apparatus}

The stimulus displays and eye monitoring were the same as those in Experiment 3, except that two identical location markers were used on each trial. The shape of the location markers varied from trial to trial. Three shapes were used: stars, diamonds, and indented rectangles (with dimensions as described in Experiment 2). Cuing was implemented as before. Targets (\#) were presented in bold Helvetica font size 32 .

\section{Procedure}

The procedures were virtually identical to those of Experiment 3. The only differences were that the location markers used within a given trial were always the same shape and all three SOAs were used. Each participant completed a practice run of 20 trials prior to completing the experimental session, which included 42 catch trials (10\% of total), 180 cued targets, and 180 uncued targets. A short break was permitted after 70 successive trials.

The variables were location cuing (cued or uncued), location marker shape (star, diamond, or rectangle), and SOA $(125,425$, or $725 \mathrm{msec}$ ), resulting in a total of 18 experimental conditions. Equal 
numbers of targets were assigned to each condition and then presented in random order. Target location was counterbalanced across experimental conditions.

\section{Results}

\section{Response Time}

Trimmed means were submitted to a 3 (location marker shape) $\times 2$ (location cuing) $\times 3$ (SOA) ANOVA. The analysis revealed significant effects of location cuing $[F(1,8)=25.32, p=.001]$ and SOA $[F(2,16)=$ $12.33, p<.001]$, as well as a significant location cuing $\times$ SOA interaction $[F(2,16)=26.38, p<.001]$. All effects replicated those of the previous experiments. ${ }^{5}$ More important, location marker shape did not significantly affect target detection RTs and did not interact with location cuing or SOA. The effects of exogenously cuing a location were the same whether targets appeared within stars, diamonds, or indented rectangles.

\section{Accuracy}

Again, target detection performance was very good. The participants accurately detected $98 \%$ of the targets and missed $1 \%$. The remaining $1 \%$ were excluded for anticipatory responses $(0.4 \%)$ and/or eye movements $(0.4 \%)$. The false alarm rate on trials in which no target was presented was less than $0.5 \%$.

\section{Discussion}

Experiment 4 showed that target detection times did not vary across the different shapes used as location markers in this study. Location marker shape did not significantly affect RT or interact with any other variable. These findings indicate that the results of Experiments 1-3 were not due to differences in the shapes used for cued objects versus uncued objects.

This finding is also consistent with the fact that the advantage for cued objects (stars) reported in Experiments 1 and 2 was specific to the $725-\mathrm{msec}$ SOA. Had targets been easier to detect within the stars, we should have observed a cued object advantage at the earlier SOAs as well. Moreover, RTs to the no-change trials of Experiment 2, in which all targets were presented in stars, did not differ from RTs to the bilateral change trials, in which no targets were presented in stars.

\section{GENERAL DISCUSSION}

The goal of the present experiments was to separate components of exogenous orienting directed to objects and to locations. To do so, we used location markers that either remained the same throughout the trial, retaining their status as cued objects or uncued objects, or changed in shape and, therefore, cuing status after being cued. This allowed us to dissociate attention effects for cued and uncued locations from those for cued and uncued objects (or shapes). The results showed significant location cuing effects at both short and long SOAs, with the typical pattern of early facilitation and later IOR. On the other hand, object cuing influenced target detection only at the longest SOA (725 msec), at which there was a facilitatory effect on RTs, the opposite effect from that observed for location cuing at the same delay. The finding of a late-onset object-based facilitation is consistent with a recent report by Ro and Rafal (1999), where object-based facilitation was observed at long SOAs in dynamic displays.

Several studies have investigated object- and locationbased orienting when the location markers or stimulus array moves after being cued (Gibson \& Egeth, 1994; Jordan \& Tipper, 1998a; Tipper \& Behrmann, 1996; Tipper et al., 1994). The results showed IOR both at the location where the cue occurred and at the new location of the cued object, suggesting that attention is reflexively oriented to both objects and locations. In our experiments, the location markers changed not in location, but in shape. The findings suggest that under these conditions, reflexive attentional orienting to objects can also be triggered by a shape change that makes that dimension salient.

We observed robust facilitation of cued locations at the short SOA across all of our experiments. Neither shape differences nor shape changes in the objects used to mark potential target locations had any effect on the magnitude of facilitation. The fact that location-based facilitation was unaltered by any of the shape changes investigated demonstrates that attention can be reflexively allocated to a designated region of space independently of the specific object represented there. In contrast, the later inhibitory component of exogenous orienting was influenced by shape changes under certain conditions. These findings are compatible with Müller and Rabbitt's (1989) conclusion that reflexive orienting effects are less susceptible to interruption at short than at long SOAs.

The data also provide evidence for reflexive orienting to cued objects at long SOAs when a shape change in the display distinguished or drew attention to the objects' shapes. The result was a benefit for cued object shapes relative to uncued object shapes at the long SOA. This advantage for cued objects occurred without cost to the other (uncued) object in the display. If cued object tokens reflexively captured attentional resources, we might have expected slower RTs on UOUL' than on UOUL trials, because only the former had a cued object in the opposite location. Yet, no such cost was ever observed.

There are two possible accounts of the fact that the benefit for cued objects was observed primarily at the cued location (facilitation for COCL, but not for COUL). One is that object-based orienting is location mediated, or contingent on attention's being allocated to the object's location. Electrophysiological studies in humans have demonstrated hierarchical processing in the visual system. In such studies, effects indicative of attention to one stimulus dimension, such as motion, color, or shape, are often observed to be contingent on the feature's being located in a relevant location (Anllo-Vento \& Hillyard, 1996; Hillyard \& Münte, 1984; see also Wijers, Mulder, Okita, $\&$ Mulder, 1989). Further evidence for the locationmediated hypothesis comes from an event-related poten- 
tial (ERP) study conducted by Weber, Kramer, and Miller (1997). Weber et al. used a modified version of Duncan's (1984) task, in which participants viewed superimposed objects (i.e., shapes) and were asked to report the presence of two features that could be located either on the same or on different objects. Typically, greater costs are observed when two features from different objects have to be reported, presumably owing to an object-based selection mechanism. Weber et al. replicated this effect and found that the amplitudes of early visual ERP components (P1 and N1) indicated differential allocation of attention in the same- versus different-object conditions. Modulations of these early ERP components are normally interpreted as reflecting spatial attention. Thus, the implication of these findings is that objects may serve to guide spatial attention, rather than providing spatially invariant representations for attentional selection.

The second account (not necessarily incompatible with the first) depends on the distinction between types and tokens. Object tokens represent particular instances of object types, maintaining their temporal and spatial continuity across motion and change (Kahneman, Treisman, \& Gibbs, 1992). If object tokens are also the units to which exogenous attention is drawn, the cued object, as operationally defined in this report, would no longer be perceived as the cued object when located in the uncued location, but simply as another object that happened to be similar in shape to the one that had been cued. Kahneman et al. showed that instances of an object are perceptually linked if the object is perceived to move, either in apparent or real motion, from one location to the next. Since there was no motion in our displays, the marker at the uncued location may have been classified as an uncued object, regardless of its similarity in shape to the cued object. The reduced or absent object cuing effects at the uncued location imply that exogenous precues do not result in reflexive orienting to cued object types. On the other hand, when an object token is seen to move through space, as in Tipper et al. (1994) and Ro and Rafal (1999), its status as a cued object is maintained.

In addition to isolating the effects of location cuing and object cuing, these studies also demonstrated a significant interaction between the two. Under circumstances in which a cued object occupied a cued location and differed from the other object in the display (unilateral COCL), object-based facilitation appeared to compete with and cancel location-based IOR at the long SOA (Figure 4C). The complete cancellation of IOR in the COCL condition (Figure 4C) further suggests that the late benefit for cued objects is of roughly the same magnitude as the costs associated with exogenous spatial cues at long SOAs. For this reason, object-based benefits may be difficult to detect under conditions in which locationbased inhibition is also present.

Experiment 3 demonstrated that the interaction between objects and locations obtained in Experiment 2 relied on the presence of a shape change in the display. In
Experiment 3, half of the trials contained two different location markers, which, after cuing, were equivalent to a cued object at the cued location (COCL condition) and an uncued object at the uncued location (UOUL) condition. It was under these same conditions in Experiments 1 and 2 that location-based IOR was eliminated for COCL targets. Nonetheless, typical IOR effects were observed in Experiment 3. The only difference in Experiment 3 was that there were no within-trial shape changes, suggesting that the shape change, rather than the presence of a differentiated cued object, was critical for evoking object-based orienting. Finally, Experiment 4 confirmed that the previous observations were not due to the specific shapes used in these studies.

One further point in relation to the object-based hypothesis is worth making. Given the reports of objectbased IOR (Gibson \& Egeth, 1994; Tipper et al., 1994) in displays containing moving or rotating objects, some object-based inhibition might have been expected in addition to the observed facilitation in the present study. Our longest SOA of $725 \mathrm{msec}$ is well within the time period in which Tipper and Weaver have observed reliable object-based IOR (see Tipper \& Weaver, 1998). One possibility is that the object-based IOR described in previous studies is really due to the updating of locations during stimulus movement. Objects in the displays used by Tipper et al. moved in synchrony along a common arc around a fixation point, so that the visual display could be grouped and perceived as rotating around this central point. With common motion linking the objects in this way, the "object-based" IOR that was observed at the cued object's new location might actually be a manifestation of location-based IOR for the same relative location within the original, albeit rotated, reference frame. By this account, object tokens play only an indirect role in IOR, by defining a frame of reference within which exogenous spatial orienting operates. Findings from one of Tipper et al.'s experiments, in which the objects provided mixed signals about the rotation of the display, are consistent with this idea. When they used displays that contained two moving boxes and two stationary boxes, no object-based IOR was observed unless the moving objects moved in front of and temporarily occluded the stationary objects. When the moving objects passed behind the stationary objects or did not overlap with the stationary objects, no object-based IOR was evident. Ro and Rafal's (1999) findings of object-based facilitation can be similarly challenged, since their objects also moved in linked fashion.

Consistent with this interpretation, Gibson and Egeth (1994) demonstrated that IOR persists at a relative location within an object when a three-dimensional object is rotated to another perspective after cuing. Their study illustrates that cued locations are defined with respect to both stimulus-defined reference frames and environmental space. In addition, Rhodes and Robertson (2001) have provided evidence that attention can be allocated 
with respect to a particular reference frame when the stimulus array is perceived to rotate following a central cue. Although they manipulated voluntary rather than reflexive attention, their results suggest the possibility that similar reference frame effects could occur in exogenous orienting. Thus, it seems possible that IOR is confined to locations and does not occur with object cuing.

\section{Conclusions}

The implications of the present findings can be summarized as follows. First, early facilitatory components of exogenous spatial orienting do not depend on location marker identity or constancy of shape. Spatial attention seems to be initially allocated to designated locations independently of the specific objects that occupy them. Second, stimulus changes in the display appeared to trigger object-based attentional orienting, producing a lateonset facilitation for cued objects relative to uncued objects at the cued location. Finally, it appears that under some circumstances, reflexive orienting to objects may compete with and even cancel the inhibition typically associated with reflexive orienting to locations at long SOAs.

\section{REFERENCES}

Anllo-Vento, L., \& Hillyard, S. A. (1996). Selective attention to the color and direction of moving stimuli: Electrophysiological correlates of hierarchical feature selection. Perception \& Psychophysics, 58, 191-206.

Barnes, L., Robertson, L. C., Beuche, A. L., \& Kim, M. (1999, April). Effects of perceived space on inhibition of return. Poster presented at the Annual Cognitive Neuroscience Society Meeting, Washington, DC.

Baylis, G. C., \& Driver, J. (1993). Visual attention and objects: Evidence for hierarchical coding of location. Journal of Experimental Psychology: Human Perception \& Performance, 19, 451-470.

Behrmann, M., \& Tipper, S. P. (1999). Attention accesses multiple reference frames: Evidence from visual neglect. Journal of Experimental Psychology: Human Perception \& Performance, 25, 83-101.

Behrmann, M., Zemel, R. S., \& Mozer, M. C. (1998). Object-based attention and occlusion: Evidence from normal participants and a computational model. Journal of Experimental Psychology: Human Perception \& Performance, 24, 1011-1036.

Buxbaum, L. J., Coslett, H. B., Montgomery, M. W., \& Farah, M. J. (1996). Mental rotation may underlie apparent object-based neglect. Neuropsychologia, 34, 113-126.

Connor, C. E., Preddie, D. C., Gallant, J. L., \& Van Essen, D. C. (1997). Spatial attention effects in macaque area V4. Journal of Neuroscience, 17, 3201-3214.

Corbetta, M., Miezin, F. M., Dobmeyer, S., Shulman, G. L., \& PeTERSEN, S. E. (1991). Selective and divided attention during visual discriminations of shape, color, and speed: Functional anatomy by positron emission tomography. Journal of Neuroscience, 11, 23832402.

DrIVER, J. (1995). Object segmentation and visual neglect. Behavioral Brain Research, 71, 135-146.

Duncan, J. (1984). Selective attention and the organization of visual information. Journal of Experimental Psychology: General, 113, 501517.

Egly, R., Driver, J., \& Rafal, R. D. (1994). Shifting visual attention between objects and locations: Evidence from normal and parietal lesion subjects. Journal of Experimental Psychology: General, 123, 161-177.
ERIKSEN, C. W., \& ST. JAmes, J. D. (1986). Visual attention within and around the field of focal attention: A zoom lens model. Perception \& Psychophysics, 40, 225-240.

FARAH, M. J. (1990). Visual agnosia. Cambridge, MA: MIT Press.

Gibson, B. S., \& EGETH, H. (1994). Inhibition of return to object-based and environment-based locations. Perception \& Psychophysics, 55, 323-339.

Hillyard, S. A., \& Münte, T. F. (1984). Selective attention to color and location: An analysis with event-related brain potentials. Perception \& Psychophysics, 36, 185-198.

Jordan, H., \& Tipper, S. P. (1998a, November). Object dimensions modulate the effect of practice on object-based IOR. Poster presented at the 39th Annual Meeting of the Psychonomic Society. Dallas, TX.

JORDAN, H., \& TIPPER, S. P. (1998b). Object-based inhibition of return in static displays. Psychonomic Bulletin \& Review, 5, 504-509.

Kahneman, D., Treisman, A., \& GibBs, B. J. (1992). The reviewing of object files: Object-specific integration of information. Cognitive Psychology, 24, 175-219.

KIM, M., \& CAVE, K. R. (1995). Spatial attention in visual search for features and feature conjunctions. Psychological Science, 6, 376-380.

Kramer, A. F., Weber, T. A., \& Watson, S. E. (1997). Object-based attentional selection-grouped arrays or spatially invariant representations? Comment on Vecera and Farah (1994). Journal of Experimental Psychology: General, 126, 3-13.

MACQuisTAN, A. D. (1997). Object-based allocation of visual attention in response to exogenous, but not endogenous, spatial precues. Psychonomic Bulletin \& Review, 4, 512-515.

Maljkovic, V., \& Nakayama, K. (1994). Priming of pop-out: I. Role of features. Memory \& Cognition, 22, 657-672.

Mangun, G. R. (1995). Neural mechanisms of visual selective attention. Psychophysiology, 32, 4-18.

Mangun, G. R., Hopfinger, J. B., Kussmaul, C. L., Fletcher, E. M., \& HeINZE, H. (1997). Covariations in ERP and PET measures of spatial selective attention in human extrastriate cortex. Human Brain Mapping, 5, 273-279.

Marshall, J. C., \& Halligan, P. W. (1993). Visuo-spatial neglect: A new copying test to assess perceptual parsing. Journal of Neurology, 240, 37-40.

MAYLOR, E. A. (1985). Facilitatory and inhibitory components of orienting in visual space. In M. I. Posner \& O. S. M. Marin (Eds.), Attention and performance XI (pp. 189-204). Hillsdale, NJ: Erlbaum.

MAYlor, E. A., \& Hockey, R. (1985). Inhibitory component of externally controlled covert orienting in space. Journal of Experimental Psychology: Human Perception \& Performance, 11, 777-787.

Mishkin, M., Ungerleider,L. G., \& Macko, K. A. (1983). Object vision and spatial vision: Two cortical pathways. Trends in Neurosciences, $\mathbf{6}, 414-417$.

Moore, C. M., Yantis, S., \& Vaughan, B. (1998). Object-based visual selection. Psychological Science, 9, 104-110.

Moran, J., \& Desimone, R. (1985). Selective attention gates visual processing in the extrastriate cortex. Science, 229, 782-784.

MülleR, H. J., \& RabBitT, P. M. A. (1989). Reflexive and voluntary orienting of visual attention: Time course of activation and resistance to interruption. Journal of Experimental Psychology: Human Perception \& Performance, 12, 315-330.

Olson, C. R., \& GetTner, S. N. (1995). Object-centered direction selectivity in the macaque supplementary eye field. Science, 269, 985-988.

Posner, M. I., \& Cohen, Y. (1984). Components of visual orienting. In H. Bouma \& D. G. Bouwhuis (Eds.), Attention and performance X: Control of language processes (pp. 531-556). Hillsdale, NJ: Erlbaum.

Posner, M. I., Rafal, R. D., Choate, L., \& Vaughan, J. (1985). Inhibition of return: Neural basis and function. Cognitive Neuropsychology, 2, 211-228.

Posner, M. I., SNyder, C. R. R., \& Davidson, B. J. (1980). Attention and detection of signals. Journal of Experimental Psychology: General, 109, 160-174.

Rhodes, D. L., \& Robertson, L. C. (2001). Endogenous spatial attention in scene-based frames of reference. Manuscript submitted for publication. 
Ro, T., \& RAFAL, R. D. (1999). Components of reflexive visual orienting to moving objects. Perception \& Psychophysics, 61, 826-836.

Robertson, L. C., \& Kim, M. (1999). Effects of perceived space on spatial attention. Psychological Science, 10, 76-79.

Robertson, L. C., Treisman, A., Friedman-Hill, S. R., \& GraboweCKy, M. (1997). The interaction of spatial and object pathways: Evidence from Balint's syndrome. Journal of Cognitive Neuroscience, 9, 254-276.

Schendel, K. L., Robertson, L. C., \& Treisman, A. (2001). Location markers in exogenous attention: Effects of their presence, duration, and offsets. Manuscript under revision.

Tassinari, G., Aglioto, S., Chelazzi, L., Peru, A., \& Berlucchi, G. (1994). Do peripheral non-informative cues induce early facilitation of target detection? Vision Research, 34, 179-189.

Tipper, S. P., \& Behrmann, M. (1996). Object-centered not scenebased visual neglect. Journal of Experimental Psychology: Human Perception \& Performance, 22, 1261-1278.

TipPer, S. P., \& WEAVER, B. (1998). The medium of attention: Locationbased, object-centered, or scene-based? In R. D. Wright (Ed.), Visual attention (pp. 77-107). New York: Oxford University Press.

Tipper, S. P., Weaver, B., Jerreat, L. M., \& Burak, A. L. (1994). Object-based and environment-based inhibition of return of visual attention. Journal of Experimental Psychology: Human Perception \& Performance, 20, 478-499.

Treisman, A. M., \& Gelade, G. (1980). A feature-integration theory of attention. Cognitive Psychology, 12, 97-136.

Treisman, A. [M.], Kahneman, D., \& Burkell, J. (1983). Perceptual objects and the cost of filtering. Perception \& Psychophysics, 6, 527532.

Ungerleider, L. G., \& HAXby, J. V. (1994). "What" and "where" in the human brain. Current Opinion in Neurobiology, 4, 157-165.

Vecera, S. P. (1994). Grouped locations and object-based attention: Comment on Egly, Driver, and Rafal (1994). Journal of Experimental Psychology: General, 123, 316-320.

VECERA, S. P. (1997). Grouped arrays versus object-based representations: Reply to Kramer et al. (1997). Journal of Experimental Psychology: General, 126, 14-18.

Vecera, S. P., \& Farah, M. J. (1994). Does visual attention select objects or locations? Journal of Experimental Psychology: General, 123, 146-160.

Weber, T. A., Kramer, A. F., \& Miller, G. A. (1997). Selective pro- cessing of superimposed objects: An electrophysiological analysis of object-based attentional selection. Biological Psychology, 45, 159182.

Wijers, A. A., Mulder, G., Okita, T., \& Mulder, L. J. (1989). Eventrelated potentials during memory search and selective attention to letter size and conjunctions of letter size and color. Psychophysiology, 26, 529-547.

Yantis, S., \& Hillstrom, A. P. (1994). Stimulus-driven attentional capture: Evidence from equiluminant visual objects. Journal of Experimental Psychology: Human Perception \& Performance, 20, 95 107.

\section{NOTES}

1. Baylis and Driver (1993) have proposed that locations are coded hierarchically so that individual parts are coded with respect to their parent object, or the more global configuration. Thus, comparing features from two different objects requires that the relative position of the two reference objects be resolved, whereas reporting two features from one object requires only one reference.

2. Owing to the final configuration of the stimulus displays, targets were $75 \%$ likely to occur in an uncued object/shape (triangle) and $25 \%$ likely to occur in a cued object (star).

3. The UOCL condition is plotted twice in Figure 2, once for the analysis of location cuing (left panels) and once for the analysis of object cuing (middle panels). Similarly, the COCL condition is plotted in the analyses of Loc + Obj cuing (right) and object cuing (middle).

4. Note that the fastest RTs were associated with targets occurring in cued objects at cued locations (COCL condition), despite the fact that targets were overall more likely to occur in uncued objects (triangles) than in cued objects (stars). Thus, cued objects seemed to benefit target detection at cued locations even though the overall probabilities favored uncued objects.

5. RTs decreased with increasing SOA and were faster for uncued targets overall (owing to IOR at two out of three SOAs). Analysis of the interaction confirmed that there was significant facilitation of cued locations at the short SOA $[F(1,16)=6.22, p<.05]$ and significant IOR at the longer SOAs $[F(1,16)=10.58$ and 60.11 , both $p$ s $<.01]$.

(Manuscript received May 9, 1998; revision accepted for publication July 24, 2000.) 